\title{
LAS BIBLIOTECAS ESCOLARES EN LA COMUNIDAD AUTÓNOMA DE GALICIA: ANÁLISIS DE LOS RECURSOS DOCUMENTALES, PERSONALES Y TECNOLÓGICOS
}

\author{
Felicidad Barreiro Fernández \\ $M^{a}$ Dolores Castro Pais \\ Elisa Teresa Zamora Rodríguez \\ $M^{a}$ Josefa Mosteiro García \\ Universidad de Santiago de Compostela
}

\begin{abstract}
RESUMEN: El presente trabajo tiene como objetivo conocer la percepción del profesorado del equipo de apoyo a la biblioteca escolar sobre los recursos documentales, personales y tecnológicos de las bibliotecas y la existencia de diferencias según el tipo de centro. Para ello, fueron seleccionados a través de un muestreo aleatorio multietápico, un total de 654 docentes del equipo de apoyo a la biblioteca escolar en la Comunidad Autónoma de Galicia a los que se les ha aplicado el Cuestionario de Evaluación del Funcionamiento de las Bibliotecas Escolares. Los resultados obtenidos revelan la existencia de diferencias en las bibliotecas escolares de los centros de educación infantil y primaria (CEIP) e institutos de educación secundaria (IES) en la adecuación de los recursos documentales a los intereses y necesidades de los usuarios y al desarrollo del currículo; en el número de horas de dedicación, funciones y necesidades de formación del equipo de apoyo a la biblioteca y en lo referente a la existencia de conexión a internet y la disponibilidad de lectores electrónicos y tabletas. De estos resultados se deriva la necesidad de llevar a cabo en las bibliotecas escolares gallegas actuaciones dirigidas a mejorar los recursos documentales, personales y tecnológicos.
\end{abstract}

PALABRAS CLAVE: biblioteca escolar, recursos, educación no universitaria, equipo de apoyo a la biblioteca escolar.

\section{SCHOOL LIBRARIES IN THE COMUNIDAD AUTÓNOMA DE GALICIA: ANALYSIS OF DOCUMENTARY, PERSONAL AND TECHNOLOGICAL RESOURCES}

\footnotetext{
ABSTRACT: The present work aims to know the perception of the teaching staff of the support team at the school library about the documentary, personal
} 
and technological resources of the libraries and the existence of differences according to the type of center. For this, a total of 654 teachers from the school library support team were selected through a multi-stage random sampling, to which the Evaluation Questionnaire of the Functioning of School Libraries has been applied. The results obtained reveal the existence of deficits in the three types of resources and differences by type of center. The results obtained reveal the existence of differences in the school libraries of the CEIP and IES in the adaptation of the documentary resources to the interests and needs of the users and to the development of the curriculum; in the number of hours of dedication, functions and training needs of the library support team and in relation to the existence of an internet connection and the availability of electronic readers and tablets. From these results derives the need to carry out actions in Galicia school libraries aimed at improving documentary, personal and technological resources.

KEYWORDS: School library, resources, non-university education, school library support team.

Recibido: $27 / 04 / 2020$

Aceptado: 16/12/2020

Correspondencia: Felicidad Barreiro Fernández, Facultad de Ciencias de la Educación, Universidad de Santiago de Compostela, Rúa Prof. Vicente Fráiz Andón, s/n, 15782 Santiago de Compostela. Email: felicidad.barreiro@usc.es

\section{INTRODUCCIÓN}

El formar a personas competentes en el uso eficiente de la información se encuentra, en la actualidad, entre los principales objetivos de la institución escolar. La Sociedad del Conocimiento y la Información necesita de una acción pedagógica distinta, no basta con proporcionar al alumnado conocimientos conceptuales ya elaborados, sino que hay que dotarlo de capacidades para analizarlos, ordenarlos y criticarlos; estableciendo un modelo educativo que implique aprender a utilizar la información. En este sentido, la biblioteca escolar es considerada el espacio propicio para esa alfabetización en información, para poner a disposición de toda la comunidad educativa el acceso libre a la información y crear situaciones y espacios de aprendizaje. Para dar respuesta a esta demanda social, nace un nuevo modelo de biblioteca escolar entendida según la Comisión Técnica de Cooperación en Bibliotecas Escolares (2015, p. 5) como:

centro de recursos de lectura, información y aprendizaje, que debe adaptar sus actuaciones a las nuevas necesidades educativas, de forma que puedan contribuir a la adquisición de las competencias básicas, apoyando las distintas áreas del currículo, así como las alfabetizaciones múltiples (lectora, mediática e informacional) de toda la comunidad escolar. 
La biblioteca escolar es un recurso y espacio fundamental en los centros educativos ya que estos tienen como misión, como ya se ha indicado, formar personas competentes en el uso eficiente de información y, para lograrlo, el tratamiento de la lectura como eje transversal resulta más necesario que nunca. Así mismo, la biblioteca juega un papel imprescindible en la implementación de planes, proyectos o programas de centro dirigidos a la adquisición de competencias básicas y hábitos de lectura, tal y como se contempla en la Ley Orgánica 2/2006, de 3 de mayo, de Educación (LOE), modificada por la Ley Orgánica 8/2013, de 9 de diciembre, para la Mejora de la Calidad Educativa (LOMCE). Su artículo 113 reconoce la necesidad de que todos los centros tengan una biblioteca escolar, entendida como un instrumento educativo que contribuya a fomentar la lectura y a que el alumnado acceda a la información y a otros recursos para el aprendizaje de las demás áreas y materias, y pueda formarse en el uso crítico de los mismos.

En esta línea, en la Comunidad Autónoma de Galicia, el fomento de la lectura y el uso de la biblioteca escolar también son considerados factores determinantes de la calidad del proceso de enseñanza aprendizaje. Tal y como se recoge en el artículo 31 de la Ley 5/2012, de 15 de junio, de bibliotecas de Galicia, las bibliotecas escolares, integradas en el sistema gallego de bibliotecas, "son recursos del centro educativo al servicio de los procesos de enseñanza y aprendizaje y del fomento de la lectura", siendo sus dos principales funciones la gestión técnica de los fondos documentales y la formación de los usuarios. En el artículo 6 de esta misma Ley se reconoce, "entre las obligaciones de las personas físicas o jurídicas titulares de las bibliotecas, la de promover en el ámbito de sus competencias, un desarrollo sostenible, coherente, innovador y constante de sus propios servicios bibliotecarios". Desde la Administración, con el objetivo de incentivar e impulsar estos nuevos proyectos de biblioteca, se convocan ayudas cada curso académico. En la última de las ayudas publicadas por la Concejalía de Educación, Universidad y Formación Profesional, regulada por la Orden del 2 de abril de 2019, por la que se convoca el Plan de mejora de bibliotecas escolares en centros públicos no universitarios para el curso 2019/20 y desarrollada por la Instrucción del 2 de septiembre de 2019, de la Dirección General de Centros y Recursos Humanos, se reconoce, entre otros aspectos, a la biblioteca como un centro de recursos de lectura, información y aprendizaje, que tiene un enfoque inclusivo y que por lo tanto contribuye a la compensación de desigualdades sociales.

Además, en el desarrollo curricular, se contempla la obligatoriedad de que todos los centros de enseñanza no universitaria elaboren sus proyectos lectores de centro, en coordinación con la persona responsable de la biblioteca, con la finalidad de articular las actuaciones de todo el profesorado, destinadas a la consecución de los objetivos pedagógicos referidos a la adquisición de la competencia lingüística y de las habilidades en el uso de la información, así como la adquisición y consolidación del hábito de la lectura. Así se recoge en el Decreto 105/2014, de 4 de septiembre y en el Decreto 133/2007, de 5 de julio, que regulan respectivamente las enseñanzas de educación primaria y de educación secundaria obligatoria en la Comunidad Autónoma de Galicia y en el Decreto 86/2015, de 25 de junio, por el que se establece el currículo de la educación secundaria obligatoria y del bachillerato en la Comunidad Autónoma de Galicia.

La biblioteca escolar se configura, de esta forma, como un recurso pedagógico al servicio del Proyecto Educativo del Centro, un elemento estratégico en el proce- 
so de enseñanza aprendizaje del alumnado, en la adquisición de hábitos lectores, en la formación y en el uso de la información etc., y por ello debe cumplir unos mínimos de calidad.

Para conocer en profundidad el funcionamiento de la biblioteca escolar, es preciso hacer una evaluación sobre la realidad que se encierra en dicho espacio, contemplando a los diferentes miembros de la comunidad educativa, entendidos como ejes clave para el mantenimiento e idónea funcionalidad de esta, así como estudiando las dimensiones que la literatura indica que se deben analizar. Según Bernal, Macías y Novoa (2011) los recursos documentales, personales y tecnológicos son, junto con otras dimensiones y elementos (la biblioteca en el centro; gestión, dinámica, uso y redes de colaboración, biblioteca y currículo; programas institucionales y valoraciones), aspectos clave si se quiere comprobar el correcto funcionamiento de las bibliotecas escolares.

En cuanto a los recursos documentales, la composición de la colección, el tipo de soportes, la cantidad de documentos existentes en la biblioteca y su procedencia son indicadores fundamentales del funcionamiento de la biblioteca. Según las directrices IFLA/UNESCO (2015) la biblioteca escolar deberá dar acceso a gran variedad de recursos físicos y digitales que permitan satisfacer las necesidades de los usuarios; incluyendo entre ellos materiales relevantes, actuales, para todas las edades, y relacionados tanto con el conocimiento y la información, como con la ficción; también deberá estar dotada de materiales audiovisuales, publicaciones periódicas o documentos informáticos en papel y/o digitales. Esto significa que:

la composición del fondo documental de una biblioteca escolar es equilibrada cuando tiene un número total de ejemplares suficiente con respecto al número total de estudiantes; cuando el porcentaje de obras de consulta y conocimiento es superior al de obras literarias o de ficción, cuando existe variedad de tipos de documentos (libros impresos, revistas, prensa, recursos electrónicos y digitales) y cuando se dispone de secciones de especial importancia tales como el fondo para a atención a la diversidad, plurilingüismo o fondo local. (Comisión Técnica de Bibliotecas Escolares, 2011, p. 27).

Por otra parte, hay que señalar que el tratamiento técnico de dicha documentación es un aspecto al que se tiene que prestar especial atención. En este sentido, la utilización de sistemas estandarizados de catalogación es de gran utilidad, facilitando el intercambio interbibliotecario y la formación de usuarios de biblioteca.

Otro aspecto determinante para un óptimo aprovechamiento de la biblioteca escolar son los recursos personales con los que cuenta. En este sentido conviene destacar que resulta imprescindible la figura de una persona responsable de la misma, cualificada y con estabilidad en el puesto de trabajo para poder coordinar, representar y encargarse de su organización y funcionamiento. El desempeño de sus funciones requiere una formación inicial contrastada y una actualización de esta, según las necesidades percibidas.

Para el desarrollo de sus tareas, la persona responsable de la biblioteca debe estar apoyada por un equipo interdisciplinar de profesores y profesoras del centro, es decir, el equipo de apoyo a la biblioteca. De ambos depende la elaboración de propuestas y el desarrollo de actividades para el adecuado funcionamiento de esta.

La posibilidad de dedicar un tiempo suficiente para realizar las funciones y tareas que le son propias, y la participación dinámica en los órganos de coordinación 
docente de la persona responsable de la biblioteca escolar y del equipo de apoyo resultan elementos clave para conseguir la implicación del profesorado del centro en el uso de los recursos disponibles en la biblioteca escolar y en el aprovechamiento de las propuestas e iniciativas de este recurso destinadas a la mejora de la acción educativa.

Los recursos tecnológicos constituyen otro de los pilares fundamentales para un óptimo aprovechamiento de la biblioteca escolar. Tal y como indican González y Faba (2013, p. 31) "para que la biblioteca escolar proporcione un adecuado aprendizaje a sus usuarios son necesarias dos condiciones: que funcionen correctamente y que no se quede al margen de las tecnologías de la información y la comunicación". El uso de las Tecnologías de la Información y de la Comunicación es fundamental para el correcto desarrollo, entre otras, de las siguientes funciones: integración de los recursos en distintos formatos y soportes, programas de alfabetización en información y alfabetización digital, nuevos servicios electrónicos o impacto en la difusión de la biblioteca.

Conocer la calidad de las bibliotecas escolares pasa, entonces, por analizar, entre otros, estos tres tipos de recursos (documentales, personales y tecnológicos) tal y como lo corroboran estudios previos (Álvarez y Cobos 1994; Baró y Mañá, 1996; Conforti y Pastoriza, 2002; Del Burgo y Bernal, 2007; Faba, 2000; Faba-Pérez y Infante-Fernández, 2018; García y Luque, 2009; Gómez, 2010; Jiménez, 2013; Marchesi y Miret, 2005; Miret, 2008; Miret, Baró, Mañá, Vellosillo y Montero, 2010; Ortiz-Repiso y Camacho, 2004; Santos, 2017). En esta misma línea, Serna, Rodríguez y Etxaniz (2017, p. 34) señalan que para apoyar el papel de las bibliotecas escolares se necesita "dotarlas de partidas presupuestarias regulares y consolidadas, de responsables especializados, de equipamientos estables y necesarios, de materiales de consulta e investigación". De ahí que en este estudio se centre la atención en el análisis de estos tres aspectos: los recursos documentales, los recursos personales y los recursos tecnológicos de las bibliotecas escolares de los centros educativos de educación infantil, primaria y secundaria sostenidos con fondos públicos en la Comunidad Autónoma de Galicia. Más concretamente, el principal objetivo del trabajo es conocer la percepción del profesorado del equipo de apoyo a la biblioteca sobre los recursos documentales, personales y tecnológicos de las bibliotecas escolares y comprobar la existencia de diferencias en función del tipo de centro.

\section{Método}

\section{Muestra}

La muestra, seleccionada a través de un muestreo aleatorio multietápico, la conforman un total de 654 docentes del equipo de apoyo a la biblioteca escolar, de ellos 365 desarrollan su labor en centros de educación infantil y primaria (CEIP) y 289 en institutos de educación secundaria (IES) sostenidos con fondos públicos de la Comunidad Autónoma de Galicia. Hay que destacar que prácticamente la totalidad del profesorado participante en el estudio, concretamente un 99.5\%, trabajan en centros de titularidad pública y la mayoría son mujeres (82.1\%). 


\section{Instrumento de recogida de datos}

Para la recogida de información se elaboró el Cuestionario de Evaluación del Funcionamiento de las Bibliotecas Escolares. La selección de las preguntas se hizo en función de la revisión documental previamente realizada y para comprobar su validez, el instrumento fue sometido a revisión por expertos de distintos ámbitos del sistema educativo entre los que se incluyen profesorado universitario y de enseñanza no universitaria y responsables de bibliotecas escolares. El cuestionario se agrupó en nueve bloques de contenido que responden a las distintas dimensiones de la biblioteca escolar recomendadas en los estudios teóricos: La biblioteca en el centro; Equipamiento, Instalaciones y Tecnologías; Recursos Documentales; Personal; Gestión; Dinámica, Uso y Redes de colaboración; Biblioteca y Currículo; Tecnologías de la Información y de la Comunicación; Programas Institucionales y Valoraciones. El cuestionario incluía también datos de identificación del centro y de identificación de la persona que contestaba.

De los nueve bloques en los que se agrupan las diferentes preguntas del cuestionario, en su mayoría de escala tipo Likert con cinco alternativas de respuesta, para este trabajo hemos analizado los aspectos referidos a los recursos documentales, personales y tecnológicos.

La fiabilidad del instrumento estimada a través del coeficiente Alpha de Cronbach fue de 0.886 con un nivel de confianza del $95.5 \%$, informando que tiene una notable consistencia interna.

El cuestionario fue enviado vía correo electrónico a los centros participantes para su distribución entre el profesorado del equipo de apoyo a la biblioteca en el año 2015.

\section{Análisis de datos}

Mediante el paquete estadístico IBM SPSS Statistics versión 24.0 se llevó a cabo un análisis descriptivo e inferencial a través del cálculo de pruebas paramétricas (t) y no paramétricas (Chi-cuadrado, U Mann-Whitney), para las preguntas referidas a los recursos documentales, personales y tecnológicos.

\section{Resultados}

A continuación, presentamos los resultados derivados del análisis de los datos recopilados mostrando, en primer lugar, aquellos referidos a los recursos documentales, continuando con los recursos personales y finalizando con los recursos tecnológicos de la biblioteca escolar.

\section{Recursos Documentales}

Respecto a la valoración de los recursos documentales de la biblioteca por parte del profesorado del equipo de apoyo a la biblioteca, en la tabla 1 se puede comprobar que existen diferencias estadísticamente significativas en seis de las once variables objeto de análisis.

En el caso de la adecuación de los fondos de la biblioteca la significación es de .002 menor que .05, por lo que se concluye que la valoración difiere significativa- 
mente en los dos grupos, siendo mayor entre el profesorado del equipo de apoyo a la biblioteca escolar de los CEIP (342.69 rango promedio). En la valoración de la actualización de los fondos de la biblioteca también se aprecia diferencia significativa ( $p=.006)$ y mayor valoración por parte del profesorado del equipo de apoyo de los CEIP (340.20 rango promedio).

Del mismo modo, el profesorado del equipo de apoyo de los CEIP considera en mayor medida que la biblioteca cuenta con materiales adecuados para el alumnado con necesidades específicas de apoyo educativo (349.87 rango promedio) y que todos los fondos registrados en el centro están en la sala de la biblioteca (338.60 rango promedio), con un nivel de significación del .000 y .002 respectivamente.

Por el contrario, pueden apreciarse diferencias estadísticamente significativas a favor del profesorado del equipo de apoyo a la biblioteca escolar de los IES (rango promedio de 340.31) en lo que respecta a si en la selección de los fondos de la biblioteca se tienen en cuenta las demandas del profesorado del equipo de apoyo a la biblioteca, con un nivel de significación de .037. Lo mismo ocurre en la valoración de la informatización de los fondos de la biblioteca, con un nivel de significación del .003 y un rango promedio de 345.90 .

Tabla 1. Diferencias por tipo de centro en la valoración de los fondos de la biblioteca escolar

\begin{tabular}{|c|c|c|c|c|c|c|}
\hline Ítems & Centro & $\mathbf{N}$ & $\begin{array}{c}\text { Rango } \\
\text { promedio }\end{array}$ & $\mathbf{U}$ & $\mathbf{Z}$ & $\mathbf{p}$ \\
\hline \multirow{2}{*}{ Los fondos son suficientes } & CEIP & 361 & 314.82 & \multirow{2}{*}{48307.500} & \multirow{2}{*}{-1.925} & \multirow{2}{*}{.054} \\
\hline & IES & 289 & 338.85 & & & \\
\hline \multirow{2}{*}{ Los fondos son adecuados } & CEIP & 361 & 342.69 & \multirow{2}{*}{45958.500} & \multirow{2}{*}{-3.108} & \multirow{2}{*}{.002} \\
\hline & IES & 289 & 304.03 & & & \\
\hline \multirow{2}{*}{ Los fondos están actualizados } & CEIP & 360 & 340.20 & \multirow{2}{*}{46189.500} & \multirow{2}{*}{-2.734} & \multirow{2}{*}{.006} \\
\hline & IES & 288 & 304.88 & & & \\
\hline \multirow{2}{*}{$\begin{array}{l}\text { Los fondos están bien } \\
\text { conservados }\end{array}$} & CEIP & 358 & 317.43 & \multirow{2}{*}{49380.000} & \multirow{2}{*}{-.958} & \multirow{2}{*}{.338} \\
\hline & IES & 286 & 328.84 & & & \\
\hline \multirow{2}{*}{$\begin{array}{l}\text { En la selección de los fondos } \\
\text { de la biblioteca se tienen } \\
\text { en cuenta las demandas del } \\
\text { profesorado del equipo de } \\
\text { apoyo a la biblioteca }\end{array}$} & CEIP & 361 & 313.64 & \multirow[b]{2}{*}{47883.000} & \multirow[b]{2}{*}{-2.088} & \multirow[b]{2}{*}{.037} \\
\hline & IES & 289 & 340.31 & & & \\
\hline
\end{tabular}




\begin{tabular}{|c|c|c|c|c|c|c|}
\hline Ítems & Centro & $\mathbf{N}$ & $\begin{array}{c}\text { Rango } \\
\text { promedio }\end{array}$ & $\mathbf{U}$ & $\mathbf{Z}$ & $\mathbf{p}$ \\
\hline \multirow{2}{*}{$\begin{array}{l}\text { La biblioteca cuenta con } \\
\text { materiales adecuados para el } \\
\text { alumnado con necesidades } \\
\text { específicas de apoyo } \\
\text { educativo }\end{array}$} & CEIP & 360 & 349.87 & \multirow[b]{2}{*}{41988.000} & \multirow[b]{2}{*}{-4.253} & \multirow[b]{2}{*}{.000} \\
\hline & IES & 286 & 290.31 & & & \\
\hline \multirow{2}{*}{$\begin{array}{l}\text { El registro de los fondos } \\
\text { informáticos existentes en el } \\
\text { centro está centralizado en la } \\
\text { biblioteca }\end{array}$} & CEIP & 357 & 322.97 & \multirow{2}{*}{49633.500} & \multirow{2}{*}{-.404} & \multirow{2}{*}{.686} \\
\hline & IES & 283 & 317.38 & & & \\
\hline \multirow{2}{*}{$\begin{array}{l}\text { Todos los fondos registrados } \\
\text { están en la sala de la } \\
\text { biblioteca }\end{array}$} & CEIP & 355 & 338.60 & \multirow{2}{*}{44160.500} & \multirow{2}{*}{-3.031} & \multirow{2}{*}{.002} \\
\hline & IES & 285 & 297.95 & & & \\
\hline \multirow{2}{*}{$\begin{array}{l}\text { Están cubiertas todas las } \\
\text { necesidades de todas las áreas } \\
\text { curriculares }\end{array}$} & CEIP & 353 & 310.01 & \multirow{2}{*}{46951.000} & \multirow{2}{*}{-1.659} & \multirow{2}{*}{.097} \\
\hline & IES & 285 & 331.26 & & & \\
\hline \multirow{2}{*}{$\begin{array}{l}\text { Los fondos están } \\
\text { informatizados }\end{array}$} & CEIP & 359 & 306.43 & \multirow{2}{*}{45388.000} & \multirow{2}{*}{-2.935} & \multirow{2}{*}{.003} \\
\hline & IES & 288 & 345.90 & & & \\
\hline \multirow{2}{*}{$\begin{array}{l}\text { Los fondos documentales } \\
\text { existentes deberían ser } \\
\text { mejorados }\end{array}$} & CEIP & 353 & 325.65 & \multirow{2}{*}{47072.500} & \multirow{2}{*}{-1.289} & \multirow{2}{*}{.198} \\
\hline & IES & 282 & 308.42 & & & \\
\hline
\end{tabular}

\section{Recursos Personales}

En relación con los recursos personales se solicitó al profesorado información respecto al número de horas semanales dedicadas a las tareas de apoyo a la biblioteca y si estas horas se encuentran fuera de las horas de docencia directa. Así mismo, se les pidió que valorasen las funciones que Ilevan a cabo como docentes del equipo de apoyo y las necesidades de formación que experimentan en diferentes ámbitos. Por último, se les preguntó acerca de la asistencia regular a actividades de formación vinculadas a las tareas que desempeñan en la biblioteca.

En lo referente al número de horas semanales dedicadas a las tareas de apoyo a la biblioteca, cabe indicar que los resultados derivados de la prueba t de Student muestran, ver tabla 2, la existencia de diferencias estadísticamente significativas entre el profesorado de los CEIP y el de los IES a favor de estos últimos que son quienes afirman dedicar mayor número de horas a la semana a las tareas de apoyo a la biblioteca $(p=.001)$. 
Tabla 2. Diferencias por tipo de centro en el número de horas semanales dedicadas a las tareas de apoyo a la biblioteca

\begin{tabular}{|c|c|c|c|c|c|c|}
\hline $\begin{array}{c}\text { Tipo de } \\
\text { Centro }\end{array}$ & $\mathbf{N}$ & Media & $\begin{array}{c}\text { Desviación } \\
\text { típica }\end{array}$ & $\mathbf{t}$ & $\mathbf{g l}$ & $\mathbf{p}$ \\
\hline CEIP & 347 & 1.78 & 1.163 & \multirow{2}{*}{-3.301} & 359.201 & .001 \\
\cline { 1 - 5 } IES & 286 & 2.38 & 2.923 & & & \\
\hline
\end{tabular}

Hay que señalar que esas horas dedicadas a las tareas de apoyo a la biblioteca se encuentran, como puede comprobarse en la figura 1, fuera de las horas de docencia directa $(79.1 \%$ en el profesorado de los IES y el $74.9 \%$ en el profesorado de los CEIP), no existiendo diferencia estadísticamente significativa entre el profesorado en función del tipo de centro $\left[x^{2}=1.541, p=.214\right]$.

Figura 1. Las horas lectivas dedicadas a las tareas de apoyo a la biblioteca están fuera de las horas de docencia directa

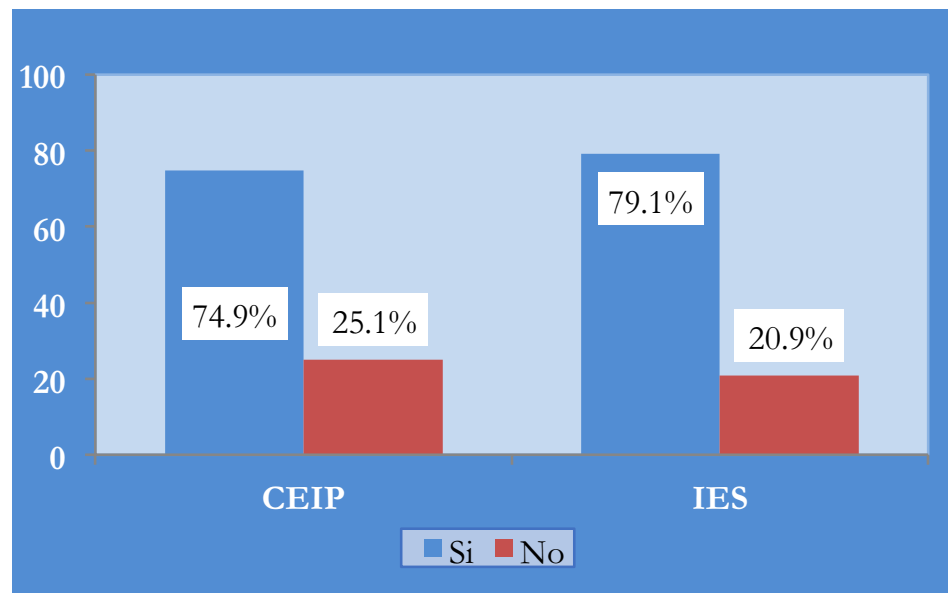

En lo referente a las funciones desarrolladas por estos profesionales, tal y como se puede observar en la tabla 3, existen diferencias estadísticamente significativas en ocho de las trece variables analizadas.

El profesorado del equipo de apoyo a la biblioteca escolar de los IES (342.41 rango promedio) afirma en mayor medida que el de los CEIP que desempeña funciones de apoyo a la persona responsable de la biblioteca en la organización y dinamización de esta, siendo esta diferencia estadísticamente significativa $(p=.029)$. Además, se han encontrado diferencias significativas en las siguientes funciones: cooperar en la organización de las actividades programadas $(p=.027)$, elaborar el Plan de Trabajo anual de la biblioteca junto con la persona responsable de la biblioteca $(p=.026)$, establecer criterios para la adquisición y actualización de los fondos de la biblioteca $(p=.013)$, recoger propuestas y sugerencias de las familias $(p<.001)$, colaborar en el 
desarrollo de la competencia lectora ( $\mathrm{p}<.001)$, colaborar en el desarrollo del hábito lector $(p<.001)$ y colaborar en el desarrollo de habilidades de trabajo con la información $(p<.001)$. La valoración de todas ellas es mayor entre el profesorado de los CEIP, es decir, el profesorado de los CEIP afirma desempeñar en mayor medida estas funciones como miembro del equipo de apoyo a la biblioteca escolar.

Tabla 3. Diferencias por tipo de centro en la valoración de las funciones como profesor/a del equipo de apoyo a la biblioteca escolar

\begin{tabular}{|c|c|c|c|c|c|c|}
\hline Ítems & Centro & $\mathbf{N}$ & $\begin{array}{c}\text { Rango } \\
\text { promedio }\end{array}$ & $\mathbf{U}$ & $\mathbf{Z}$ & $\mathbf{p}$ \\
\hline \multirow{2}{*}{$\begin{array}{l}\text { Apoyar a la persona } \\
\text { responsable de la biblioteca } \\
\text { en la organización y } \\
\text { dinamización }\end{array}$} & CEIP & 362 & 312.05 & \multirow[b]{2}{*}{47259.000} & \multirow[b]{2}{*}{-2.177} & \multirow[b]{2}{*}{.029} \\
\hline & IES & 288 & 342.41 & & & \\
\hline \multirow{2}{*}{$\begin{array}{l}\text { Recopilar información, } \\
\text { materiales y recursos } \\
\text { necesarios para el } \\
\text { funcionamiento de la } \\
\text { biblioteca }\end{array}$} & CEIP & 361 & 323.82 & \multirow[b]{2}{*}{51327.000} & \multirow[b]{2}{*}{-0.51} & \multirow[b]{2}{*}{.959} \\
\hline & IES & 285 & 323.09 & & & \\
\hline \multirow{2}{*}{$\begin{array}{l}\text { Cooperar en el diseño de las } \\
\text { actividades programadas }\end{array}$} & CEIP & 359 & 335.86 & \multirow{2}{*}{47080.500} & \multirow{2}{*}{-1.989} & \multirow{2}{*}{.047} \\
\hline & IES & 287 & 308.04 & & & \\
\hline \multirow{2}{*}{$\begin{array}{l}\text { Cooperar en la organización } \\
\text { de las actividades } \\
\text { programadas }\end{array}$} & CEIP & 363 & 339.14 & \multirow{2}{*}{47140.000} & \multirow{2}{*}{-2.209} & \multirow{2}{*}{.027} \\
\hline & IES & 287 & 308.25 & & & \\
\hline \multirow{2}{*}{$\begin{array}{l}\text { Elaborar el Plan de Trabajo } \\
\text { anual de la biblioteca junto } \\
\text { con la persona responsable } \\
\text { de la biblioteca }\end{array}$} & CEIP & 357 & 335.54 & \multirow[b]{2}{*}{45860.500} & \multirow[b]{2}{*}{-2.225} & \multirow[b]{2}{*}{.026} \\
\hline & IES & 285 & 303.91 & & & \\
\hline \multirow{2}{*}{$\begin{array}{l}\text { Cooperar en la puesta en } \\
\text { marcha de las actividades } \\
\text { programadas en la biblioteca }\end{array}$} & CEIP & 362 & 336.09 & \multirow{2}{*}{47572.000} & \multirow{2}{*}{-1.888} & \multirow{2}{*}{.059} \\
\hline & IES & 286 & 309.84 & & & \\
\hline \multirow{2}{*}{$\begin{array}{l}\text { Establecer criterios para la } \\
\text { adquisición y actualización } \\
\text { de los fondos de la biblioteca }\end{array}$} & CEIP & 357 & 337.01 & \multirow{2}{*}{45336.000} & \multirow{2}{*}{-2.487} & \multirow{2}{*}{.013} \\
\hline & IES & 285 & 302.07 & & & \\
\hline \multirow{2}{*}{$\begin{array}{l}\text { Recoger propuestas y } \\
\text { sugerencias del profesorado }\end{array}$} & CEIP & 357 & 332.37 & \multirow{2}{*}{46991.500} & \multirow{2}{*}{-1.735} & \multirow{2}{*}{.083} \\
\hline & IES & 285 & 307.88 & & & \\
\hline \multirow{2}{*}{$\begin{array}{l}\text { Recoger propuestas y } \\
\text { sugerencias del alumnado }\end{array}$} & CEIP & 351 & 323.09 & \multirow{2}{*}{47703.000} & - 898 & 369 \\
\hline & IES & 283 & 310.56 & & -.090 & \\
\hline Recoger propuestas y & CEIP & 357 & 367.90 & 31807500 & -8010 & 000 \\
\hline sugerencias de las familias & IES & 278 & 253.92 & & & \\
\hline Colaborar en el desarrollo de & CEIP & 358 & 355.10 & 0 & 5507 & ل \\
\hline la competencia lectora & IES & 283 & 277.87 & 30450.300 & -5.592 & .000 \\
\hline
\end{tabular}




\begin{tabular}{|c|c|c|c|c|c|c|}
\hline Ítems & Centro & $\mathbf{N}$ & $\begin{array}{c}\text { Rango } \\
\text { promedio }\end{array}$ & $\mathbf{U}$ & $\mathbf{Z}$ & $\mathbf{p}$ \\
\hline \multirow{2}{*}{$\begin{array}{l}\text { Colaborar en el desarrollo del } \\
\text { hábito lector }\end{array}$} & CEIP & 360 & 352.20 & \multirow{2}{*}{40789.000} & \multirow{2}{*}{-4.811} & \multirow{2}{*}{.000} \\
\hline & IES & 285 & 286.12 & & & \\
\hline \multirow{2}{*}{$\begin{array}{l}\text { Colaborar en el desarrollo de } \\
\text { habilidades de trabajo con la } \\
\text { información }\end{array}$} & CEIP & 359 & 345.08 & \multirow{2}{*}{42333.500} & \multirow{2}{*}{-3.828} & \multirow{2}{*}{.000} \\
\hline & IES & 283 & 291.59 & & & \\
\hline
\end{tabular}

En relación con la asistencia regular a actividades de formación vinculadas a las tareas como docente del equipo de apoyo a la biblioteca, en la figura 2, puede apreciarse que un 55.9\% del profesorado de secundaria indica asistir regularmente a este tipo de formación mientras que sólo lo afirma el $41.3 \%$ del profesorado de los CEIP. En la prueba chi-cuadrado la significación es de .000, por lo que se concluye que existen diferencias estadísticamente significativas según el tipo de centro en la asistencia regular a actividades de formación $\left[x^{2}=13.588, p<.001\right]$.

Figura 2. Asistencia regular a actividades de formación vinculadas a las tareas como profesorado del equipo de apoyo a la biblioteca

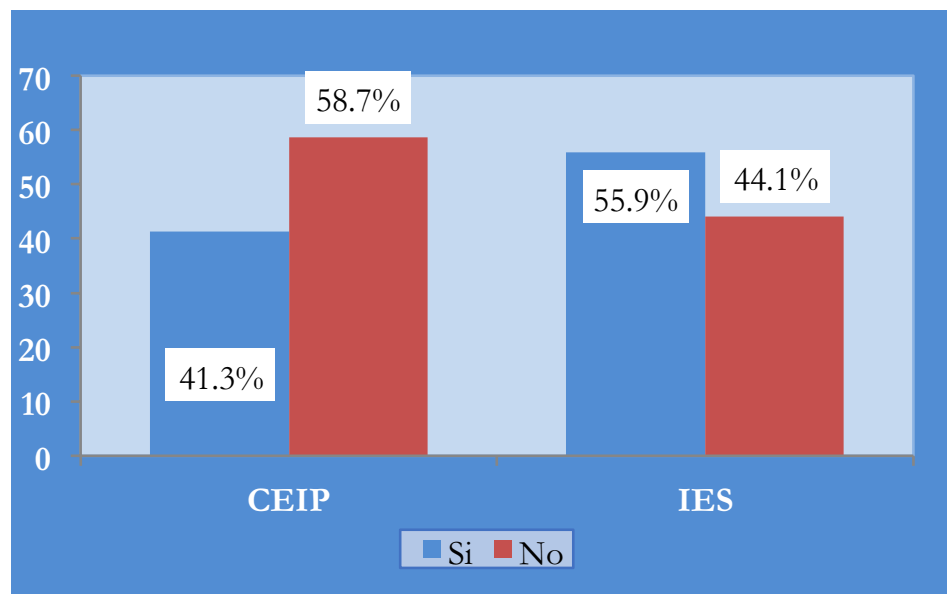

Siguiendo con la temática de la formación se preguntó al profesorado acerca de la necesidad de formación en diferentes ámbitos para poder desarrollar las tareas de apoyo a la biblioteca; en la tabla 4 podemos comprobar la existencia de diferencias estadísticamente significativas en cuatro de las siete variables objeto de análisis.

En el caso de la necesidad de formación en biblioteconomía la significación es de .035, por lo que se concluye que la valoración difiere significativamente en los dos grupos, siendo mayor entre el profesorado del equipo de apoyo a la biblioteca escolar de los CEIP (326.99 rango promedio). En cuanto a la necesidad de formación en lectura y literatura infantil y juvenil se aprecia diferencia significativa $(p=.000)$ y mayor valoración por parte del profesorado del equipo de apoyo de los CEIP (341.33 
rango promedio). Así mismo, el profesorado del equipo de apoyo de los CEIP valora en mayor medida la necesidad de recibir formación en alfabetización en información (330.23 rango promedio) y en trabajo por proyectos (332.15 rango promedio), con un nivel de significación del .014 y .009 respectivamente.

Tabla 4. Diferencias por tipo de centro en la valoración de las necesidades de formación

\begin{tabular}{|c|c|c|c|c|c|c|}
\hline Ítems & Centro & $\mathbf{N}$ & $\begin{array}{c}\text { Rango } \\
\text { promedio }\end{array}$ & $\mathbf{U}$ & $\mathbf{Z}$ & $\mathbf{p}$ \\
\hline \multirow{2}{*}{ Biblioteconomía } & CEIP & 349 & 326.99 & \multirow{2}{*}{43977.000} & \multirow{2}{*}{-2.108} & \multirow{2}{*}{.035} \\
\hline & IES & 278 & 297.69 & & & \\
\hline \multirow{2}{*}{$\begin{array}{l}\text { Lectura y Literatura } \\
\text { Infantil y Juvenil }\end{array}$} & CEIP & 353 & 341.33 & \multirow{2}{*}{41891.500} & \multirow{2}{*}{-3.823} & \multirow{2}{*}{.000} \\
\hline & IES & 283 & 290.03 & & & \\
\hline \multirow{2}{*}{$\begin{array}{l}\text { Alfabetización en } \\
\text { Información }\end{array}$} & CEIP & 352 & 330.23 & \multirow{2}{*}{43742.000} & \multirow{2}{*}{-2.464} & \multirow{2}{*}{.014} \\
\hline & IES & 278 & 296.85 & & & \\
\hline \multirow{2}{*}{$\begin{array}{l}\text { Tecnologías de la } \\
\text { información y la } \\
\text { comunicación }\end{array}$} & CEIP & 357 & 329.93 & \multirow{2}{*}{47863.000} & \multirow{2}{*}{-1.430} & \multirow{2}{*}{.153} \\
\hline & IES & 285 & 310.94 & & & \\
\hline \multirow{2}{*}{ Formación de usuarios } & CEIP & 358 & 331.48 & \multirow{2}{*}{46904.000} & \multirow{2}{*}{-1.762} & \multirow{2}{*}{.078} \\
\hline & IES & 283 & 307.74 & & & \\
\hline \multirow{2}{*}{$\begin{array}{l}\text { Gestión y } \\
\text { Dinamización }\end{array}$} & CEIP & 356 & 326.36 & \multirow{2}{*}{48467.500} & \multirow{2}{*}{-.986} & \multirow{2}{*}{.324} \\
\hline & IES & 284 & 313.16 & & & \\
\hline \multirow{2}{*}{ Trabajo por proyectos } & CEIP & 356 & 332.15 & \multirow{2}{*}{43558.000} & \multirow{2}{*}{-2.628} & \multirow{2}{*}{.009} \\
\hline & IES & 276 & 296.32 & & & \\
\hline
\end{tabular}

\section{Recursos Tecnológicos}

En lo referente a los recursos tecnológicos se preguntó al profesorado del equipo de apoyo a la biblioteca escolar por la existencia de conexión a internet y red wifi en la biblioteca, la valoración del servicio de internet, el número de ordenadores disponibles con acceso a internet y la existencia de lectores electrónicos o tabletas. Para finalizar se les solicitó que valorasen la adecuación del número de ordenadores con que cuenta la biblioteca.

En la figura 3 se puede observar que el profesorado del equipo de apoyo a la biblioteca de los IES es el que más afirma que existe acceso a internet en la biblioteca $(98.9 \%)$ frente al $93.6 \%$ del profesorado de los CEIP, siendo esta diferencia estadísticamente significativa $\left[\mathrm{x}^{2}=11.596, \mathrm{p}=.001\right]$. 
Figura 3. Disponibilidad de acceso a internet

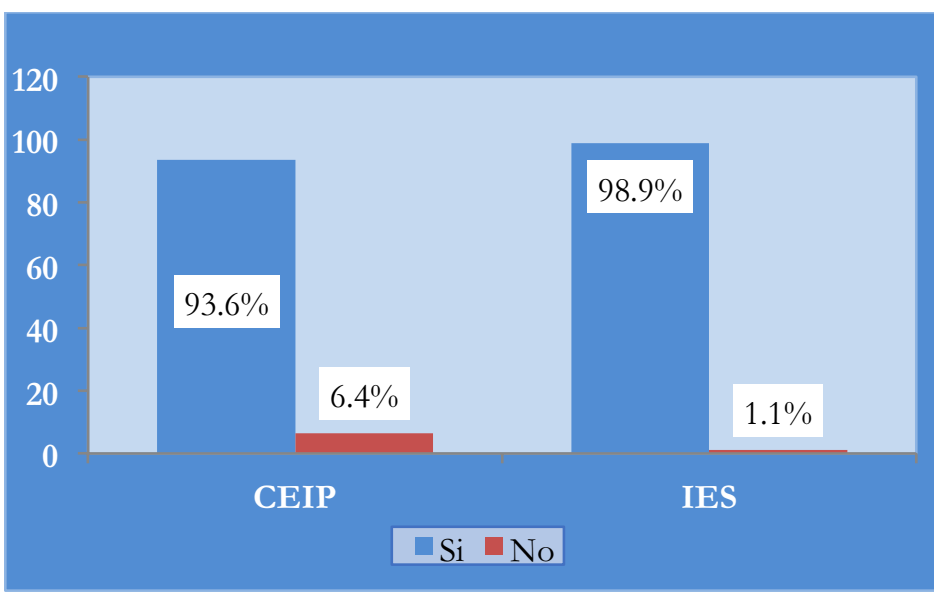

Según el profesorado del equipo de apoyo a la biblioteca, ver figura 4, un $81.3 \%$ de las bibliotecas de los CEIP frente al $67.4 \%$ de las de los IES tienen red wifi. En la prueba chi-cuadrado el nivel de significación es .000, lo que lleva a concluir que existen diferencias significativas según el tipo de centro $\left[x^{2}=16.172, p<.001\right]$.

Figura 4. Disponibilidad de red wifi

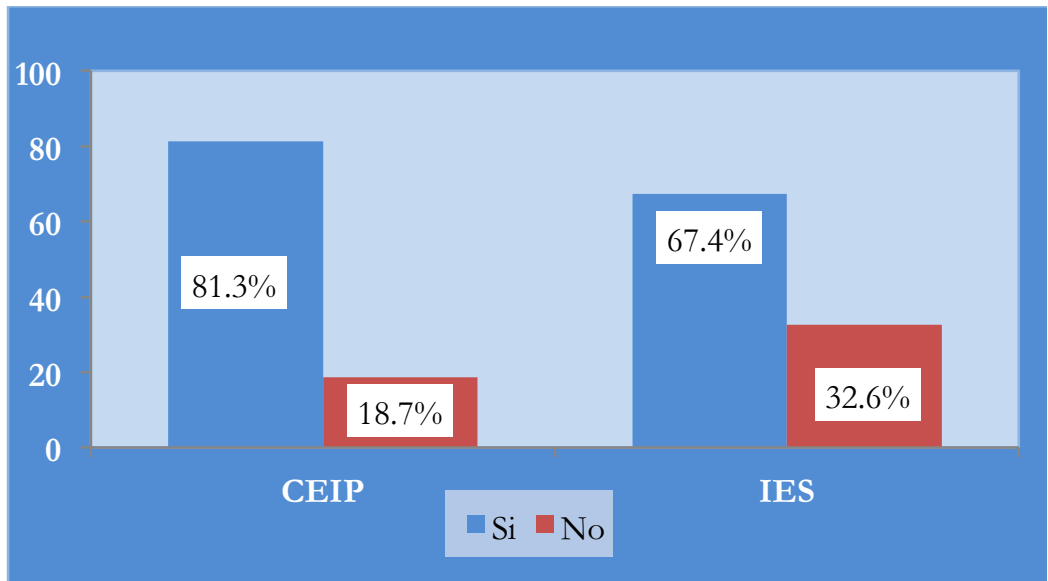

En la valoración del servicio de internet de la biblioteca escolar indicar que no existen diferencias significativas por tipo de centro, tal y como se puede comprobar en la tabla 5. 
Tabla 5. Diferencias por tipo de centro en la valoración del grado de adecuación del servicio de internet de la biblioteca

\begin{tabular}{|l|c|c|c|c|c|c|}
\hline Ítems & Centro & $\mathbf{N}$ & $\begin{array}{c}\text { Rango } \\
\text { promedio }\end{array}$ & $\mathbf{U}$ & $\mathbf{Z}$ & $\mathbf{p}$ \\
\hline $\begin{array}{l}\text { El acceso a internet de la } \\
\text { biblioteca escolar }\end{array}$ & CEIP & 357 & 331.53 & 47647.500 & -1.523 & .128 \\
\cline { 2 - 8 } & IES & 286 & 310.10 & \\
\hline
\end{tabular}

En cuanto al número de ordenadores disponibles con acceso a internet, indicar que los resultados de la prueba $t$ de Student muestran la existencia de diferencias estadísticamente significativas, tal y como se contempla en la tabla 6, a favor de las bibliotecas de los IES $\left(\mathrm{t}_{607}=-.6 .614, \mathrm{p}<.001\right)$.

Tabla 6. Diferencias por tipo de centro en el número de ordenadores disponibles con acceso a internet

\begin{tabular}{|c|c|c|c|c|c|c|}
\hline Tipo de Centro & $\mathbf{N}$ & Media & Desviación típica & $\mathbf{t}$ & gl & p \\
\hline CEIP & 337 & 4.24 & 3.126 & \multirow{2}{*}{-6.614} & \multirow{2}{*}{607} & \multirow{2}{*}{.000} \\
\hline IES & 272 & 5.90 & 3.037 & & & \\
\hline
\end{tabular}

En la figura 5 se puede apreciar que el profesorado del equipo de apoyo a la biblioteca de los IES es el que más afirma que en la biblioteca se cuenta con lectores electrónicos o tabletas (42.2\%) frente al 31.7\% del profesorado de los CEIP, siendo esta diferencia estadísticamente significativa $\left[x^{2}=7.296, p=.007\right]$.

Figura 5. Disponibilidad de lectores electrónicos o tabletas

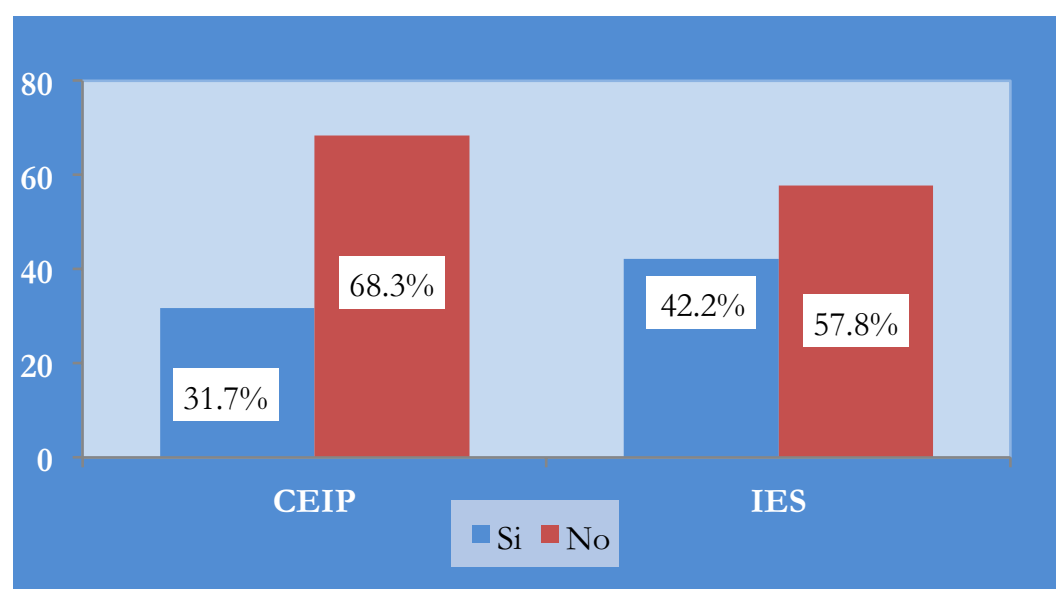

Finalmente, indicar que no existen diferencias significativas por tipo de centro en la valoración del grado de adecuación del número de ordenadores de la biblioteca escolar. 
Tabla 7. Diferencias por tipo de centro en la valoración del grado de adecuación del número de ordenadores de la biblioteca escolar

\begin{tabular}{|l|c|c|c|c|c|c|}
\hline Ítems & Centro & $\mathbf{N}$ & $\begin{array}{c}\text { Rango } \\
\text { promedio }\end{array}$ & $\mathbf{U}$ & $\mathbf{Z}$ & $\mathbf{p}$ \\
\hline $\begin{array}{l}\text { Número de ordenadores de la } \\
\text { biblioteca escolar }\end{array}$ & CEIP & 361 & 316.90 & 49059.500 & -1.211 & .226 \\
\cline { 2 - 4 } & IES & 287 & 334.06 & \\
\hline
\end{tabular}

\section{Discusión}

Para el buen funcionamiento de las bibliotecas escolares resulta imprescindible disponer de recursos documentales, personales y tecnológicos, sólo así se pueden diseñar y elaborar actuaciones ajustadas a las necesidades y demandas específicas de los distintos miembros de la comunidad educativa. A este respecto, en el estudio realizado se constatan unos mejores resultados, a nivel global, en cuanto al análisis de los recursos documentales, adecuados a los intereses y necesidades de los usuarios y a los diversos niveles de aprendizaje, en relación con los datos que refleja el estudio de Baró, Mañá y Comalat (2002, p. 78) que indica "la existencia de fondos obsoletos y poco adecuados a los intereses y necesidades del alumnado y a los diversos niveles de aprendizaje" y el de Miret et al. (2013) que constata que siguen existiendo déficits en los materiales de conocimiento y apoyo al currículo, tanto impresos como electrónicos y, también, en relación al uso de la biblioteca como apoyo al desarrollo curricular de las diferentes materias y la calidad de las obras existentes sobre diferentes materias no literarias, que en el caso del estudio de García y Picó (2015) se presenta como deficitario.

Aunque cabe indicar que los resultados muestran, igualmente, diferencias significativas por parte del profesorado del equipo de apoyo a la biblioteca escolar de los CEIP y de los IES en relación con las variables analizadas. Por una parte, los miembros de los CEIP consideran que los fondos son adecuados, están actualizados, registrados en la sala de la biblioteca y cuentan con materiales adecuados para el alumnado con necesidades específicas de apoyo educativo. Pero a pesar de esto indican que los fondos existentes deberían ser mejorados y por su parte el profesorado del equipo de apoyo a la biblioteca escolar de los IES señala que los fondos son suficientes, están bien conservados e informatizados, y que para su selección se tienen en cuenta sus demandas, estando cubiertas todas las necesidades de todas las áreas curriculares. Estos resultados son similares a los obtenidos en otros estudios realizados sobre la temática, tales como el de Camacho y Ortiz-Repiso (2005); Miret et al. (2013) y Santos (2017) que constatan, en relación con los recursos documentales, que han mejorado; señalando, también un afianzamiento en la provisión de fondos.

En lo referente a los recursos personales, los resultados obtenidos ponen de manifiesto, también, diferencias significativas entre el profesorado del equipo de apoyo a la biblioteca escolar de los CEIP y de los IES en relación con las variables analizadas. En concreto, el profesorado de los IES afirma dedicar mayor número de horas a la semana a las tareas de apoyo a la biblioteca que el profesorado de los CEIP, horas que, en ambos casos, se realizan al margen de la docencia directa.

En cuanto a las funciones desarrolladas, cabe indicar que en la misma medida, tanto el profesorado del equipo de apoyo a la biblioteca escolar de los CEIP como 
el de los IES recopila información, materiales y recursos necesarios para el funcionamiento de la biblioteca, además, entre ellos existen diferencias significativas en relación a otras funciones, ya que en mayor medida el profesorado del equipo de apoyo a la biblioteca escolar de los IES apoya a la persona responsable de la biblioteca en la organización y dinamización de la misma y, por su parte, el profesorado del equipo de apoyo a la biblioteca escolar de los CEIP coopera en el diseño de actividades programadas, en su organización y puesta en marcha, elabora el Plan de Trabajo anual junto con la persona responsable de la biblioteca, recoge propuestas y sugerencias del profesorado, alumnado y familias, así como establece criterios para la adquisición y actualización de los fondos de la biblioteca y colabora en el desarrollo de la competencia lectora, del hábito lector y de habilidades de trabajo con la información.

En relación con la formación, elemento fundamental para el puesto que desempeñan, igualmente se observan diferencias significativas según el tipo de centro, ya que, si bien el profesorado asiste de manera regular a actividades de formación vinculadas a las tareas como profesorado de equipo de apoyo a la biblioteca, esta asistencia es más regular entre el profesorado de secundaria que entre el profesorado de los CEIP. Este resultado está relacionado con las necesidades de formación que señalan los profesores y profesoras de los CEIP. Entre dichas demandas de formación destacan la vinculada con la formación en biblioteconomía, en lectura y literatura infantil y juvenil, alfabetización en información y en trabajo por proyectos. Podemos concluir que se observan resultados similares a los de los estudios de Camacho y Ortiz-Repiso (2005); Baró et al. (2002) y García y Picó (2015) que muestran una baja valoración en cuanto a la formación, indicando todos ellos como punto débil la escasa formación del personal que atiende las bibliotecas escolares.

Por último, en lo referente a los recursos tecnológicos indicar que se observan diferencias entre los tipos de centros en relación a la existencia de conexión a internet y disponibilidad de lectores electrónicos o tabletas en la biblioteca en el caso de los IES y red wifi en el caso de los CEIP. Sin embargo, no se aprecian diferencias significativas en cuanto a la valoración del servicio de internet y el grado de adecuación del número de ordenadores y del acceso a internet de la biblioteca escolar. Resultados coincidentes con los obtenidos por Baró et al. (2002); Camacho y Ortiz (2005) y Miret et al. (2013) que señalan que en los centros se van integrando los recursos tecnológicos y que las dotaciones en las bibliotecas van aumentando de forma progresiva.

En base a los resultados obtenidos, se observa que las bibliotecas escolares gallegas se "están enfrentando a un proceso de transformación orientado a mejorar sus equipamientos, recursos y servicios, y colaborar así en la mejora de la calidad de la enseñanza" (Novoa, 2007, p. 93); y desde esta perspectiva sería recomendable llevar a cabo actuaciones dirigidas a mejorar los fondos documentales, principalmente de los CEIP, incrementar el número de funciones en la biblioteca escolar, al profesorado de los IES, así como fomentar la participación del equipo de apoyo a la biblioteca en los CEIP en actividades de formación que mejoren sus necesidades formativas, apostando por una implantación y uso eficaz de los recursos tecnológicos ya que "nada es más adecuado que una biblioteca plenamente integrada con las nuevas tecnologías de la información y la comunicación" (Conforti y Pastoriza, 2002, p. 41) y de esta forma se podrá "superar el concepto de la biblioteca como espacio donde se 
fomenta únicamente la lectura para ir más allá y considerarla como un espacio donde se lee, se investiga, se colabora y se innova" (Comisión Técnica de Cooperación en Bibliotecas Escolares, 2015, p. 5).

\section{ReFERENCIAS BIBLIOGRÁFICAS}

Álvarez, M. y Cobos, L. (1994). La biblioteca escolar en España: problemas para su normalización. En J. A. Gómez Hernández (Coord.), Lectura, Educación y Bibliotecas: ideas para crear buenos lectores (pp. 33-52). Murcia: ANABAD.

Baró, M. y Mañá, T. (1996). Formarse para informarse. Madrid: Celeste.

Baró, M., Mañá, T. y Comalat, M. (2002). Las bibliotecas de los centros públicos de educación secundaria en la ciudad de Barcelona. Anales de Documentación, 5, 51-79. http://revistas.um.es/analesdoc/article/view/2231

Bernal, A. I., Macías, C. y Novoa, C. (Coords.) (2011). Marco de referencia para las bibliotecas escolares. Madrid: Ministerio de Educación, Secretaria de Estado de Educación y Formación Profesional, Dirección General de Evaluación y Cooperación Territorial Subdirección General de Cooperación Territorial.

Camacho, J. A. y Ortiz-Repiso, V. (2005). Las bibliotecas escolares en CastillaLa Mancha y sus usuarios. Estudio de la situación durante el curso 20022003, Educación y biblioteca, 146, 118-133. https://dialnet.unirioja.es/servlet/ articulo?codigo=1128766

Comisión Técnica de Bibliotecas Escolares (2011). Marco de referencia para las bibliotecas escolares. Madrid: Ministerio de Educación. http://www.ccbiblio.es/wpcontent/uploads/Marcoreferenciabescolares.pdf

Comisión Técnica de Cooperación en Bibliotecas Escolares (2015). Informe noviembre 2015, Madrid: Consejo de Cooperación Bibliotecaria. http://www.ccbiblio.es/ wp-content/uploads/InformeCTC_B_EscolaresPleno2016.pdf

Conforti, N. y Pastoriza, N. E. (2002). Las nuevas tecnologías y la biblioteca escolar. Educación y biblioteca, 130, 38-43. http://hdl.handle.net/10366/118862

Decreto 133/2007, de 5 de julio, que regulan las enseñanzas de educación secundaria obligatoria en la Comunidad Autónoma de Galicia. Diario Oficial de Galicia, 13 de julio de 2007, nº. 136, pp. 12.032-12.199. http://sid.usal.es/idocs/F3/ LYN14655/14655-eso.pdf

Decreto 105/2014, de 4 de septiembre, por el que se establece el currículo de educación primaria en la Comunidad Autónoma de Galicia. Diario Oficial de Galicia, 9 de septiembre de 2014, no.171, pp. 37406-38087. https://www.xunta.gal/dog/ Publicados/2014/20140909/AnuncioG0164-050914-0005_es.pdf

Decreto 86/2015, de 25 de junio, por el que se establece el currículo de la educación secundaria obligatoria y del bachillerato en la Comunidad Autónoma de Galicia. Diario Oficial de Galicia, 29 de junio de 2015, n. 120, pp. 25.434-27.073. http://www.mecd.gob.es/dms/mecd/educacion-mecd/mc/lomce/mapa-ccaa/ bachillerato/galicia-curriculo-eso-bach/galicia-curriculo-eso-bach.pdf

Del Burgo, M. A. y Bernal, A. I. (2007). Las bibliotecas escolares navarras: 1999-2006. Pamplona: Gobierno de Navarra. 
Faba, C. (2000). Las bibliotecas escolares y Extremadura: un estilo comparativo nacional e internacional. Boletín de la ANABAD, 50(2), 119-134.

Faba-Pérez, C. y Infante-Fernández, L.M. (2018). The content disseminated on social media by public secondary school libraries as a reflection of society: The case of the Extremadura region of Spain. The Electronic Library, 37(1), 16-34. https://doi. org/10.1108/EL-04-2018-0073

García, J. y Picó, M. T. (2015). Bibliotecas escolares en la provincia de Valencia. Métodos de información, MEI, II, 6(11), 175-200. http://doi.org/10.5557/IIMEI6N11-175200

García, J. y Luque, J. M. (2009). Estudio sobre el desarrollo del Plan de Lectura y de Bibliotecas Escolares en la provincia de Málaga. Curso 2008/2009 (Separata de Libro Abierto, 37). http://www.juntadeandalucia.es/educacion/webportal/abacoportlet/content/24665a56-f4e1-49e1-aba3-dea988ea2c46

Gómez, J. A. (2010). Las bibliotecas escolares en España ante una nueva década: brotes verdes e incertidumbres. Anuario ThinkEPI, 1, 94-102.

González-Mateos, I. y Faba-Pérez, C. (2013). Modelos para evaluar la situación de las bibliotecas escolares y la calidad de sus sitios web. Investigación Bibliotecológica: archivonomía, bibliotecología e información, 28(63), 29-50.

IFLA/UNESCO (2015). Directrices de la IFLA/UNESCO para la biblioteca Escolar (2 ${ }^{\text {a }}$ ed.). https://www.ifla.org/publications/node/9512

Instrucción del 2 de septiembre de 2019, de la Dirección General de Centros y Recursos Humanos en relación con la organización y el funcionamiento de las bibliotecas escolares, durante el curso 2019/2020, en los centros docentes de niveles no universitarios, de titularidad de la Consellería de Educación, Universidad y Formación Profesional. https://www.edu.xunta.gal/portal/sites/web/ files/dxcrrhh_instrucions_biblitoeca_escolar_curso19_20.pdf

Jiménez, C. M. (2013). Estudio sobre el estado de las webs de bibliotecas escolares en Andalucía y Extremadura y propuestas para su mejora. Investigación bibliotecológica, 27(60), 27-50.

Ley 5/2012, de 15 de junio, de bibliotecas de Galicia. Diario Oficial de Galicia, 27 de junio de 2012, no 122, pp. 25.553-25.579. https://www.xunta.gal/dog/ Publicados/2012/20120627/AnuncioC3B0-200612-0001_es.pdf

Ley Orgánica 2/2006, de 3 de mayo, de Educación. Boletín Oficial de Estado, 4 de mayo de 2006, no 106, pp. 17.158-17.207. https://www.boe.es/boe/dias/2006/05/04/ pdfs/A17158-17207.pdf

Ley Orgánica 8/2013, de 9 de diciembre, para la Mejora de la Calidad Educativa. Boletín Oficial de Estado, 10 de diciembre de 2013, n. 295, pp. 97.858-97.921. https://www.boe.es/boe/dias/2013/12/10/pdfs/BOE-A-2013-12886.pdf

Marchesi, A. y Miret, I. (2005). Las Bibliotecas escolares en España: análisis y recomendaciones. Salamanca: Fundación Germán Sánchez Ruipérez.

Miret, I. (2008). Bibliotecas escolares (aún más) hoy. En J. A. Millán (Coord.), La lectura en España: Informe 2008: Leer para aprender (pp. 93-105). Madrid: Federación de Gremios de Editores de España y Fundación Germán Sánchez Ruipérez.

Miret, I., Baró, M., Mañá, T., Vellosillo, I. y Montero, I. (2010). Bibliotecas escolares "entre comillas". Estudio de casos: buenas prácticas en la integración de la biblioteca 
en los centros educativos. Madrid: Fundación Germán Sánchez Ruipérez. http:// www.fundaciongsr.com/uploads/contenidos/doc/122-2BE\%20entre_comillas.pdf

Miret, I., Baró, M., Mañá, T. y Vellosillo, I. (2013). Las bibliotecas escolares en España. Dinámicas 2005-2011. Madrid: Fundación Germán Sánchez Ruipérez. https://sede.educacion.gob.es/publiventa/las-bibliotecas-escolares-en-espanadinamicas-2005-2011/educacion-bibliotecas/16078

Novoa, C. (2007). Bibliotecas Escolares de Galicia tejiendo redes de colaboración a favor de la educación, Educación y biblioteca, 161, 93-97. https://dialnet.unirioja. es/servlet/articulo?codigo=2374442, 2007.

Orden de 2 de abril de 2019 por la que se convoca el Plan de mejora de bibliotecas escolares en centros públicos no universitarios de titularidad de esta consellería para el curso 2019/20. Diario Oficial de Galicia, 16 de abril de 2019, $\mathrm{n}^{\circ} 74$, pp. 18923-1896. https://www.xunta.gal/dog/Publicados/2019/20190416/ AnuncioG0534-080419-0003_es.html

Ortiz-Repiso, V. y Camacho, J. A. (2004). Las bibliotecas escolares de Castilla-La Mancha: Análisis y situación actual: Curso 2002-2003. Toledo: Junta de Comunidades de Castilla-La Mancha, Consejería de Educación y Ciencia.

Santos, I. C. (2017). Desarrollo curricular y recursos educativos en las bibliotecas escolares, Investigaciones Sobre Lectura, 7, 36-54. https://www. comprensionlectora.es/revistaisl/index.php/revistalSL/article/view/196

Serna, M., Rodríguez, A. y Etxaniz, X. (2017). Biblioteca escolar y hábitos lectores en los escolares de Educación Primaria. Ocnos, 16(1), 18-49. http://doi.org/10.18239/ ocnos_2017.16.1.1205 